\title{
Can echocardiographically estimated pulmonary arterial elastance be a non-invasive predictor of pulmonary vascular resistance?
}

Neeraj Sinha', Srikala Devabhaktuni², Aparna Kadambi³, John A. McClung', Wilbert S. Aronow ${ }^{2,4}$, Stuart G. Lehrman"

\author{
'Division of Pulmonary and Transplantation Medicine, Department of Medicine, \\ The Methodist Hospital, Houston, TX, USA \\ ${ }^{2}$ Division of Cardiology, Department of Medicine, Westchester Medical Center, \\ New York Medical College, Valhalla, NY, USA \\ ${ }^{3}$ Department of Medicine, Westchester Medical Center, New York Medical College, \\ Valhalla, NY, USA \\ ${ }^{4}$ Division of Pulmonary, Critical Care, and Sleep Medicine, Department of Medicine, \\ Westchester Medical Center, New York Medical College, Valhalla, NY, USA
}

Submitted: 17 August 2013

Accepted: 10 November 2013

Arch Med Sci 2014; 10, 4: 692-700

DOI: $10.5114 /$ aoms.2014.44860

Copyright @ 2014 Termedia \& Banach

\section{Abstract}

Introduction: Measurement of pulmonary vascular resistance (PVR) is essential in evaluating a patient with pulmonary hypertension.

Material and methods: Data from right heart catheterization (RHC) and echocardiograms performed within 90 days of each other on 45 non-consecutive adult patients were reviewed in this retrospective study. Patients were recruited using an assortment of strategies to ensure the presence of patients with a wide range of PVR.

Results: The linear regression equation between RHC-derived PVR and echocardiographic pulmonary arterial elastance $(\mathrm{PAE})$ was: $\mathrm{PVR}=(562.6 \times \mathrm{PAE})$ - $38.9(R=0.56, p<0.0001)$. An adjustment for echocardiographic PAE was made by multiplying it by hemoglobin (in $\mathrm{g} / \mathrm{dl}$ ) and (right atrial area) ${ }^{1.5}$ (in $\mathrm{cm}^{3}$ ). As RHC-derived PVR varies with blood hemoglobin, an adjustment for PVR was made for hemoglobin of $12 \mathrm{~g} / \mathrm{dl}$. Visualization of the XY scatter plot of adjusted PVR and adjusted PAE isolated a subset of patients with PVR higher than 8.8 Wood units, where a strong linear relationship existed (adjusted PVR $=(0.89 \times$ adjusted PAE $)+137.4, R=0.89, p=0.008)$.

Conclusions: The correlation coefficient of the regression equation connecting echocardiographic PAE and RHC-derived PVR was moderate. In a subset of patients with very high PVR and after appropriate adjustment, a strong linear relationship existed with an excellent correlation coefficient.

Key words: pulmonary vascular resistance, pulmonary arterial elastance, echocardiography.

\section{Introduction}

Measurement of pulmonary vascular resistance (PVR) is important in evaluating pulmonary hypertension (PH). The PVR derived by right heart catheterization (RHC) is regarded as the gold standard. However, as RHC is invasive and may be infeasible in some patients, non-invasive indices of PVR are needed. Non-invasive indices should distinguish with high

\author{
Corresponding author: \\ Wilbert S. Aronow MD, FACC, \\ FAHA \\ Cardiology Division \\ New York Medical College \\ Macy Pavilion, Room 138 \\ Valhalla, NY 10595, USA \\ Phone: (914) 493-5311 \\ Fax: (914) 235-6274 \\ E-mail:wsaronow@aol.com
}


accuracy the patients with low PVR from those with high PVR. Also, they should be precise over a broad range of PVR. Such non-invasive indices may help in assessing vasodilator reactivity and in monitoring treatment response.

The following indices among others have been used to predict PVR by using linear regression equations [1, 2]: [SPAP/(HR $\left.\left.\times \mathrm{VTI}_{\text {RVOT }}\right)\right]$ and TR$\mathrm{JV}_{\text {PEAK }} / \mathrm{VTI}_{\text {RVOT}}$, where SPAP - systolic pulmonary arterial pressure, $\mathrm{HR}$ - heart rate, $\mathrm{VTI}_{\text {RVOT }}$ - velocity time integral of flow through right ventricular outflow tract; and TRJV PEAK $_{-}$- peak tricuspid regurgitant jet velocity. Equations derived from these indices have moderate correlation coefficients. However, a linear equation may not work if units on two sides of the equation are vastly different. Compared to the unit of dyne $\times \mathrm{s} / \mathrm{cm}^{5}$ for PVR, the unit is dyne $/\left(\mathrm{s} \times \mathrm{cm}^{3}\right)$ for $\left[\mathrm{SPAP} /\left(\mathrm{HR} \times \mathrm{VTI}_{\mathrm{RVOT}}\right)\right]$ and $\mathrm{s}^{-1}$ for peak TRJV/VTI ${ }_{\text {RVOT }}$. A polynomial equation might be a better approach. Or, a predictor with a unit similar to that of PVR would be more appropriate.

Pulmonary arterial elastance (PAE) is defined as pulmonary artery pulse pressure (PP) divided by stroke volume (SV). The PP is the difference between SPAP and DPAP (diastolic pulmonary artery pressure). Units of PAE include dyne $/ \mathrm{cm}^{5}$ and $\mathrm{mm} \mathrm{Hg} / \mathrm{ml}$. We hypothesized that echocardiographic PAE may be a superior predictor of PVR compared to the aforesaid indices, and may provide a linear equation which remains accurate over a wide range of PVR.

The aim of this retrospective study was to assess the relationship between echocardiographic PAE and RHC-derived PVR values (by obtaining an $X Y$ scatterplot of those values, and performing appropriate regression analyses between them).

\section{Material and methods}

\section{Study population}

Five hundred and forty-eight patients underwent RHC from April 1, 2011 until March 31, 2012. Every third patient on the list of 548 patients (which was in the alphabetical order of the last name) was retrospectively evaluated on the electronic medical records for the presence of echocardiographic and RHC data. Our target number of patients to screen was 183. If echocardiographic data on a patient were missing on the electronic medical records (as they might have been performed at an outside facility), that patient was excluded. Other exclusion criteria were: 1) performance of cardiac surgery between the dates of echocardiography and RHC, 2) presence of data indicative of initiation of pulmonary vasodilators between the dates of echocardiography and RHC, 3) lack of adequate tricuspid valve and/ or pulmonary valve regurgitation jets, presence of an intracardiac shunt, presence of severe tricuspid regurgitation or pulmonary regurgitation, presence of pulmonic stenosis, or presence of more than mild aortic insufficiency or mitral regurgitation, 4) lack of cardiac output obtained by the Fick method on RHC - as we intended to use only Fick method derived PVR for our study), 5) use of mechanical ventilation/inotropes/pressors at the time of performance of echocardiography or RHC, and 6) the difference between echocardiography and RHC performance dates being greater than 90 days. Out of 183 patients, only 37 could be included due to the exclusion criteria mentioned in the above paragraph.

We anticipated that the vast majority of patients in the above-mentioned list would have normal to moderately high PVRs, although an ideal method of selection would involve studying all consecutive patients over a time period, and hoping that patients with all ranges of PVRs in adequate numbers would fall in that group. However, patients who have higher PVR are relatively few due to rarity of the diseases having this state. In order to include more patients with high PVR for the final analysis, we used the following strategies: 1) We contacted the pulmonary hypertension specialist practicing in our hospital to provide a list of the patients who were on treatment for pulmonary arterial hypertension. Out of 11 such patients, only 5 could be included due to the exclusion criteria mentioned in the above paragraph. 2) We screened for any additional patients with high PVR (greater than 200 dyne $\times \mathrm{s} / \mathrm{cm}^{5}$ ) from a list of inpatients who were admitted from April 1, 2011 until March 31, 2012 and had the discharge diagnosis ICD-9 codes of 416.0 and 416.8. ICD-9 codes 416.0 and 416.8 stand for primary pulmonary hypertension and other chronic pulmonary heart diseases, respectively. Out of 126 patients, only 5 had high PVR. Out of those 5 patients, only 1 additional patient could be included due to the exclusion criteria mentioned in the above paragraph. 3) We obtained a list of inpatients from the Department of Pharmacy in the foresaid period who underwent treatment with a pulmonary hypertension medication (epoprostenol, treprostinil, bosentan, ambrisentan, and sildenafil), and the list was screened for any additional patients with high PVR (greater than 200 dynes $\times \mathrm{s} / \mathrm{cm}^{5}$ ). Out of 12 such patients, only 2 could be included due to the exclusion criteria mentioned in the above paragraph.

When multiple echocardiograms and/or RHCs had been performed during the study period on any patient, only one pair of echocardiogram and RHC which was closest together in duration was selected. No patient was included twice in the final list. The final list had 45 patients. 


\section{Measurements}

The following formulae were used to compute PAE (simplified modified Bernoulli equation was used where needed): SPAP $=4 \times\left(T_{\text {RJV }}\right)_{\text {PEAK }}{ }^{2}+\operatorname{RAP}(\mathrm{i})$ $\left(\mathrm{RAP}-\right.$ right atrial pressure); DPAP $=4 \times\left(\mathrm{PRJV}_{\mathrm{ED}}\right)^{2}$ + RAP (ii) $\left(P R J V_{E D}=\right.$ pulmonary valve regurgitant jet velocity at end diastole); and PP = SPAP - DPAP $=4\left[\left(\text { TRJV }_{\text {PEAK }}\right)^{2}-\left(\text { PRJV }_{\text {ED }}\right)^{2}\right]$ (iii).

\section{Equation (iii) eliminates the need for estimating RAP}

Assuming there is no intracardiac shunt, SV from the right ventricle (RV) and left ventricle (LV) are expected to be the same as RV and LV are in series. Left ventricular outflow tract (LVOT) flow velocity profile using pulse wave Doppler is almost always obtained in the echocardiograms performed in our hospital. The RVOT flow velocity envelopes, if obtained, are obtained by continuous wave Doppler, which would not be as accurate as the ones obtained by pulse wave Doppler. Due to the above-mentioned reasons, we used the following formula for SV [3]: SV $=\mathrm{VTI}_{\text {LVOT }} \times$ cross-Sectional area of LVOT (iv). Thus, PAE $=\mathrm{PP} / \mathrm{SV}=$ $\left(4\left[\left(\text { TRJV }_{\text {PEAK }}\right)^{2}-\left(\text { PRJV }_{\text {ED }}\right)^{2}\right]\right) /\left(V_{T I} I_{\text {LVOT }} \times\right.$ cross-sectional area of LVOT) (v).

The echocardiographic estimation of a pressure gradient between RV and RA by using a simplified Bernoulli equation (as in equations (i) and (ii)) may be affected by the following two phenomena. Firstly, there is a well-described pressure recovery effect in which inertial forces cause some of the pressure to recover after the passage through a narrow valve. Thus, true pressure gradient is reduced [4]. An RHC measurement would pick up this true pressure gradient. However, an echocardiographic estimation based on a simplified Bernoulli equation (utilizing TRJV $_{\text {PEAK }}$ ) would overestimate the pressure gradient. Inertial forces are greater when the volume of the receiving chamber (in this case, the right atrium) is smaller [4]. Secondly, the simplified Bernoulli equation ignores the viscosity factor - which is a part of the unabridged Bernoulli equation. Viscosity of blood is most strongly influenced by its hemoglobin concentration $[5,6]$. When the viscosity factor is high, using a simplified Bernoulli equation would result in underestimation of the true pressure gradient. We adjusted the simplified Bernoulli equation derived pressure gradient by multiplying it by a marker for viscosity and by right atrial (RA) volume. We took blood hemoglobin level $(\mathrm{Hb})$ as the marker for viscosity [7]. The largest RA cross-sectional area was measured on an echocardiogram, and the RA was assumed to be spherical. Thus, the volume was calculated using equation (vi) derived as follows:
RA volume $=(4 / 3) \times \pi \times(\text { RA radius })^{3}=(4 / 3) \times$ $\pi \times(\text { RA cross-sectional area } / \pi)^{3 / 2}$ [Cross-sectional

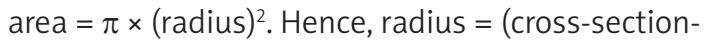
al area $\left./ \pi)^{1 / 2}\right]$. Thus, RA volume $=[(4 / 3) /(\sqrt{ } \pi)] \times($ RA cross-sectional area) $)^{3 / 2}$ (vi).

As the Pearson coefficient of correlation remains unaffected if all the values on one particular axis are changed in the same proportion [8], we dropped the $[(4 / 3) /(\sqrt{ } \pi)]$ factor. Thus, the adjusted echocardiographically estimated pressure gradient between two chambers was: adjusted pressure gradient $=$ (echocardiographically estimated pressure gradient) $\times \mathrm{Hb} \times($ RA cross-sectional area) $)^{3 / 2}$ (vii).

The unit on the right hand side of equation (vii) remains the same as those for pressure despite this adjustment, as using equation (vii) is akin to adjusting the echocardiographically estimated pressure to a hypothetical patient with $1 \mathrm{~g} / \mathrm{dl}$ of blood $\mathrm{Hb}$ and $1 \mathrm{~cm}^{3}$ of RA volume. Thus, the units of $\mathrm{Hb}$ and $\mathrm{RA}$ volume cancel out in equation (vii).

As the above-mentioned viscosity adjustment factor is the same for SPAP and DPAP, and if we ignore the effect of pressure recovery phenomenon for smaller DPAP, the equation for adjusted PAE may be derived from solving (iii) and (iv): adjusted $\mathrm{PAE}=\mathrm{PAE} \times \mathrm{Hb} \times(\text { RA cross-sectional area) })^{3 / 2}$ (viii).

The PVR as measured by RHC is also affected by $\mathrm{Hb}$. Resistance as per Poiseuille's law is directly proportional to the viscosity of the fluid. In the case of blood, the predominant factor that determines the viscosity is $\mathrm{Hb}$. Thus, a patient with a similar degree of 'pulmonary vascular disease' will have different RHC-derived PVRs, if $\mathrm{Hb}$ is different [9]. We adjusted all RHC measured PVRs for our final analysis to $\mathrm{Hb}$ of $12 \mathrm{~g} / \mathrm{dl}$, using the following equation [10]: adjusted PVR $=[P V R \times$ $273.28] /\left[1+(5.05 \times \mathrm{Hb})+\left(1.47 \times \mathrm{Hb}^{2}\right)\right](\mathrm{ix})$.

Based on equation (ix), in a hypothetical patient, an RHC-measured PVR of 200 dyne $\times \mathrm{s} / \mathrm{cm}^{5}$ at $\mathrm{Hb}$ of $8 \mathrm{~g} / \mathrm{dl}$ will change to about 600 dyne $\times \mathrm{s} / \mathrm{cm}^{5}$ if $\mathrm{Hb}$ increases to $15 \mathrm{~g} / \mathrm{dl}$, assuming all other variables remain unchanged. The nature of the relationship on linear regression analysis, the Pearson coefficient of correlation, and the $p$ value would not have changed if any other $\mathrm{Hb}$ had been used [8].

All echocardiographic measurements, based on American Society of Echocardiography guidelines, were made by a physician who was blinded to the results of RHC. Two-dimensional Doppler echocardiography was performed using the Philips iE33 ultrasound system (Philips Medical Systems, Andover, MA). Continuous-wave Doppler was used to obtain the TRJV Doppler gain was appropriately adjusted to avoid overestimation or underestimation of maximal velocities. The highest velocity obtained from multiple views was used. Flow velocity envelope of the 
left ventricular outflow tract (LVOT) was obtained by pulse wave Doppler. Velocity timed integral of LVOT $\left(\mathrm{VTI}_{\text {LVOT }}\right)$ was obtained from LVOT flow velocity envelope. Diameter of LVOT was obtained from parasternal long axis view as per the ASE guidelines. In patients with atrial fibrillation, the average of three measurements was used. The largest right atrial cross-sectional area was obtained from the apical four chamber view. Right atrial pressure (RAP) was measured as per American Society of Echocardiography recommendations [11]. All measurements were made by a physician (SD), who was blinded to the results of RHC.

The study was approved by the Institutional Review Board at our hospital (Westchester Medical Center, Valhalla, NY - approval number L-10,457).

\section{Statistical analysis}

The GraphPad Prism 6.0 software package was used for the statistical analysis. Linear regression was done when appropriate. Pearson coefficient of correlation was calculated when appropriate. One-way ANOVA was used for continuous variables, and $\chi^{2}$ test for multiple groups was used for categorical variables. A $p$ value less than 0.05 was considered significant.

\section{Results}

Baseline characteristics are described in Table I. The median interval between the paired echocardiogram and RHC was 1 day, with a range of 0-75 days. A scatter plot demonstrating the relationship between PAE and PVR is shown in Figure 1. The Pearson correlation coefficient was moderate, but statistically significant: PVR $=(562.6 \times \mathrm{PAE})+$ $38.9(R=0.56, p<0.0001)(\mathrm{x})$.

A scatter plot of PVR adjusted for $\mathrm{Hb}\left(\mathrm{PVR}_{\mathrm{adj}}\right)$ and echocardiographic PAE adjusted for $\mathrm{Hb}$ and RA size $\left(P A E_{a d j}\right)$ is shown in Figure 2. The Pearson coefficient of correlation was low, but statistically significant): $\mathrm{PVR}_{\mathrm{adj}}=0.098 \times \mathrm{PAE}_{\mathrm{adj}}+295.4(R=$ $0.41, p=0.0048)(x i)$.

However, a visual analysis of the scatter plot (Figure 2) revealed broadly two sets of data points. The set of 7 data points demarcated by the top bracket appeared to have a strong linear relationship. The set of 37 data points demarcated by the bottom bracket appeared to have no relationship. One particular data point (as depicted by the arrow) was an extreme value. The extreme value was due to an extremely large RA cross-sectional area of $54 \mathrm{~cm}^{2}$. A preliminary visual analysis of a scatter plot to explore the nature of the relationship and to explore whether more than one relationship might exist is a necessary statistical practice [8]. Also, it is recommended to further analyze data after pruning rare extreme values [12].
We separately analyzed the set of 7 data points in the top bracket and the set of 37 data points in the bottom bracket.

A scatter plot of the top 7 data points is shown in Figure 3. The Pearson correlation coefficient was excellent: $\mathrm{PVR}_{\mathrm{adj}}=0.89 \times \mathrm{PAE}_{\mathrm{adj}}+137.4(R=$ $0.89, p=0.008)(\mathrm{xii})$.

A scatter plot of the bottom 37 data points is shown in Figure 4 . The Pearson correlation coefficient was poor: $\mathrm{PVR}_{\text {adj }}=0.036 \times \mathrm{PAE}_{\text {adj }}+221.9(R=$ $0.14, p=0.468)$ (xiii).

Inter-observer variability analysis for the echocardiographic measurements was done. Ten echocardiograms from the study were randomly chosen. One of the authors (JAM) blinded to all findings reviewed the following measurements again: TRJV $\mathrm{PEAK}_{\text {, }}$ PRJV $\mathrm{ED}_{\text {ED }}, \mathrm{VTI}_{\text {LVOT }}$, and diameter of LVOT. As these variables are continuous, Pearson coefficients of correlation $(R)$ were calculated to assess inter-observer variability. $R$ values were $0.98,0.76,0.88$, and 0.71 for TRJV $_{\text {PEAK, }}$ PRJV $\mathrm{ED}_{\text {, }}$ $\mathrm{VTI}_{\mathrm{LVOT}}$, and diameter of LVOT, respectively.

\section{Discussion}

We found a strong linear relationship between PVR (adjusted for $\mathrm{Hb}$ of $12 \mathrm{~g} / \mathrm{dl}$ ) and echocardiographic PAE (adjusted for hemoglobin and RA size), when $P R_{\text {adj }}$ was very high $\left[P V R_{\text {adj }}>706\right.$ dyne-s/cm $\mathrm{cm}^{5}$ (8.8 Wood units)]. We hypothesized the following explanation for our result. Measurement of PVR ignores the crucial variable of closing pressure of pulmonary vessels. The Starling resistor model best describes the pressure gradient driving the flow in the easily distensible/collapsible pulmonary vessels (Figure 2). Due to the effects of alveolar pressure (for vessels lying close to alveoli) and intrapleural pressure (for bigger extra-alveolar vessels), pulmonary vessels may close at a pressure higher than the left atrial pressure (top diagram in Figure 5). Flow (Q) is determined by the gradient between an inflow pressure, or mean pulmonary artery pressure (MPAP), and an outflow pressure which is either closing pressure $\left(P_{c}\right)$ or left atrial pressure $\left(P_{1 a}\right)$. When $P_{1 a}$ is greater than $P_{c}$, the MPAP versus $Q$ relationship crosses the origin (line A) and PVR is constant [bottom left and right diagrams respectively]. When $P_{c}$ is greater than $P_{1 a}$, the MPAP versus $Q$ relationship has a positive pressure intercept (line B) and PVR decreases in a hyperbolic fashion with increasing Q [bottom left and right diagrams respectively]. Closing pressure cannot be measured during the routine RHC. Without the knowledge of the closing pressure, PVR becomes an erroneous and inconsistent marker of the presence or severity of pulmonary vascular disease of pre-capillary origin.

Pulmonary arteries and arterioles in pulmonary vascular diseases of pre-capillary origin 


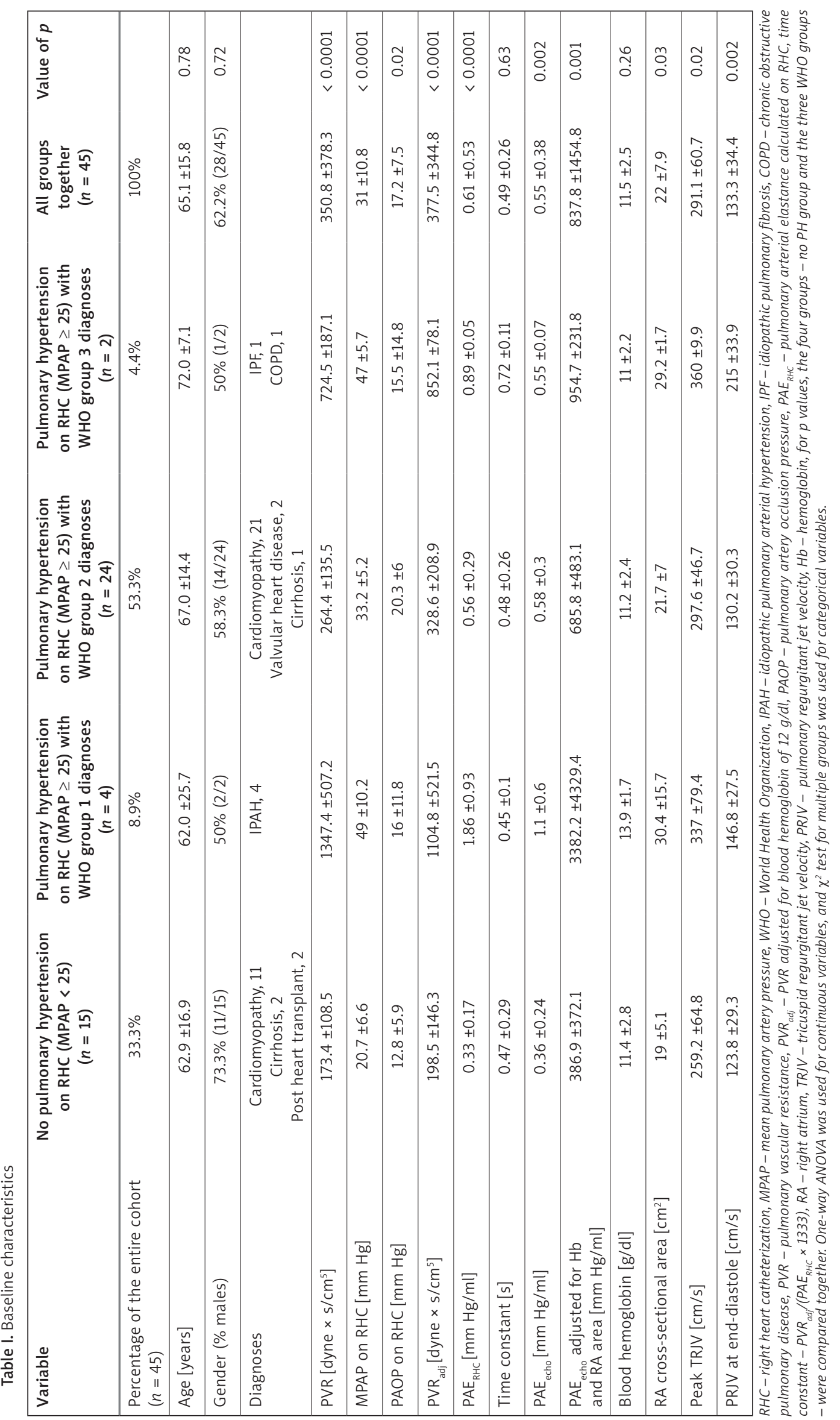




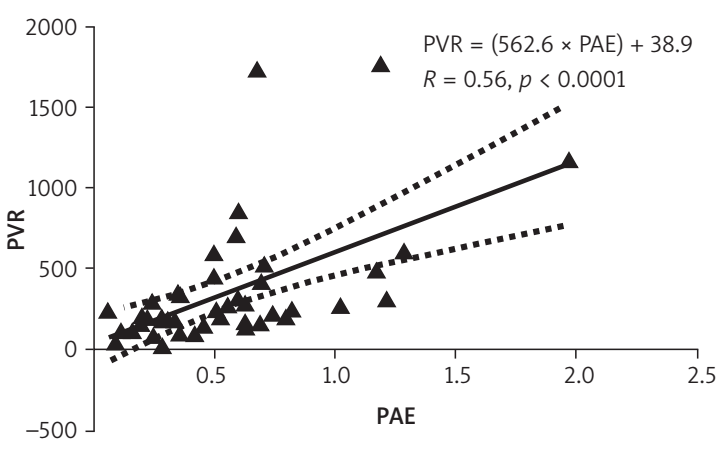

Figure 1. Linear regression analysis between PVR and PAE

PVR - pulmonary vascular resistance, PAE - pulmonary arterial elastance. Units for $P V R$ : dyne $\times \mathrm{s} / \mathrm{cm}^{5}$. Units for PAE: $\mathrm{mm} \mathrm{Hg} / \mathrm{ml}$. $R$ - Pearson coefficient of correlation

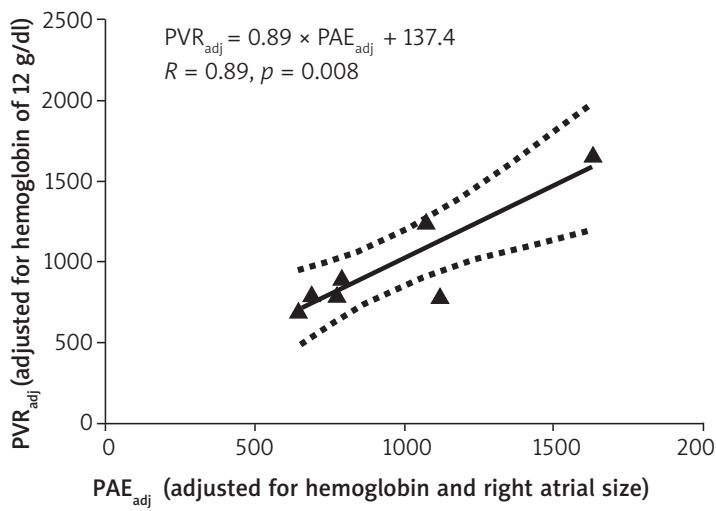

Figure 3. Linear regression analysis between $P V R_{\text {adj }}$ and $P A E_{\text {d.: }}$ Only the data points as depicted within the top brackets in Figure 2 are included. Lowest $P V R_{\text {adj }}$ in this small data set corresponds to 706.0 dyne-s/ $\mathrm{cm}^{5}$

$P V R_{\text {at }}$ - pulmonary vascular resistance for hemoglobin of $12 \mathrm{~g} /$ dl (see text for details). Units for $P V R_{\text {adj }}$ : dyne $\times \mathrm{s} / \mathrm{cm}^{5}$. PAE - adjusted echocardiographic pulmonary

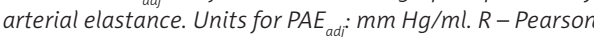
coefficient of correlation

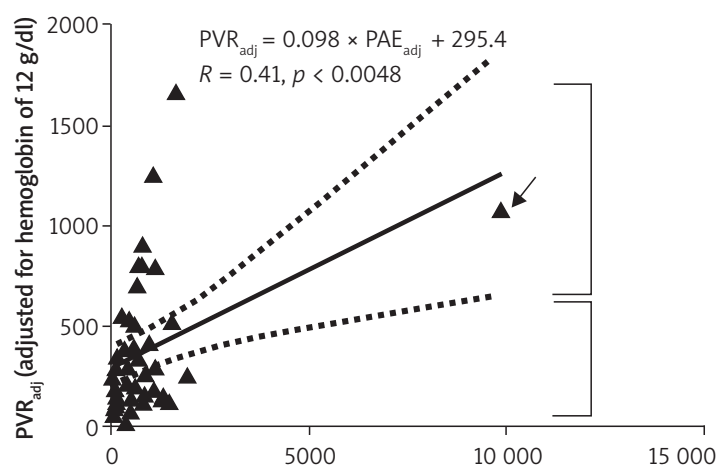

$\mathrm{PVR}_{\mathrm{adj}}$ (adjusted for hemoglobin and right atrial size)

Figure 2. Linear regression analysis between $\mathrm{PVR}_{\mathrm{adj}}$ and $P R_{\text {adj: }}$ See text for the description of data points depicted by the arrow and the two square brackets

$P V R_{a d j}$ - pulmonary vascular resistance for hemoglobin of $12 \mathrm{~g} / \mathrm{dl}$ (see text for details). Units for PVR : dyne $\times$ $\mathrm{s} / \mathrm{cm}^{5}$. PAE $\mathrm{s}_{\text {adj }}$ - adjusted echocardiographic pulmonary arterial elastance. Units for $P A E_{a d i} \mathrm{~mm} \mathrm{Hg} / \mathrm{ml}$. R-Pearson coefficient of correlation

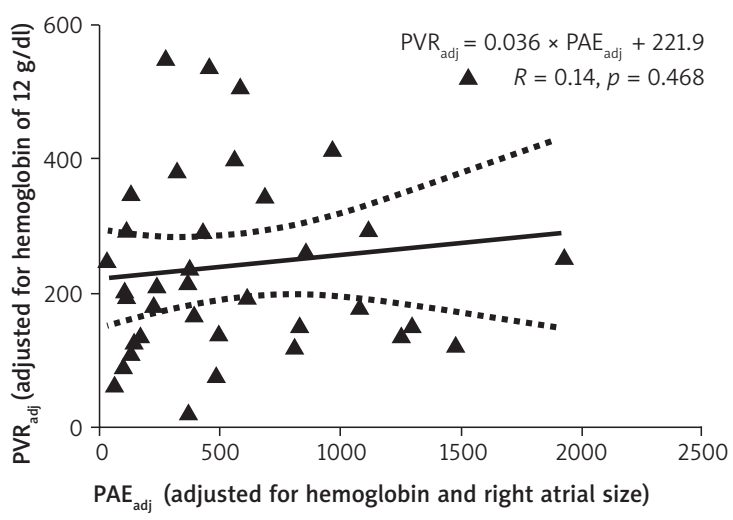

Figure 4. Linear regression analysis between $P V R_{\text {adj }}$ and $\mathrm{PAE}_{\mathrm{adj}}$. Only the data points as depicted within the bottom brackets in Figure 2 are included

$P V R_{a d j}$ - pulmonary vascular resistance for hemoglobin of $12 \mathrm{~g} / \mathrm{dl}$ (see text for details). Units for $P V R_{\text {adi }}$ dyne $\times$ $\mathrm{s} / \mathrm{cm}^{5}$. PAE arterial elastance. Units for $P A E_{\text {adj }} \mathrm{mm} \mathrm{Hg} / \mathrm{ml} . R$ - Pearson coefficient of correlation
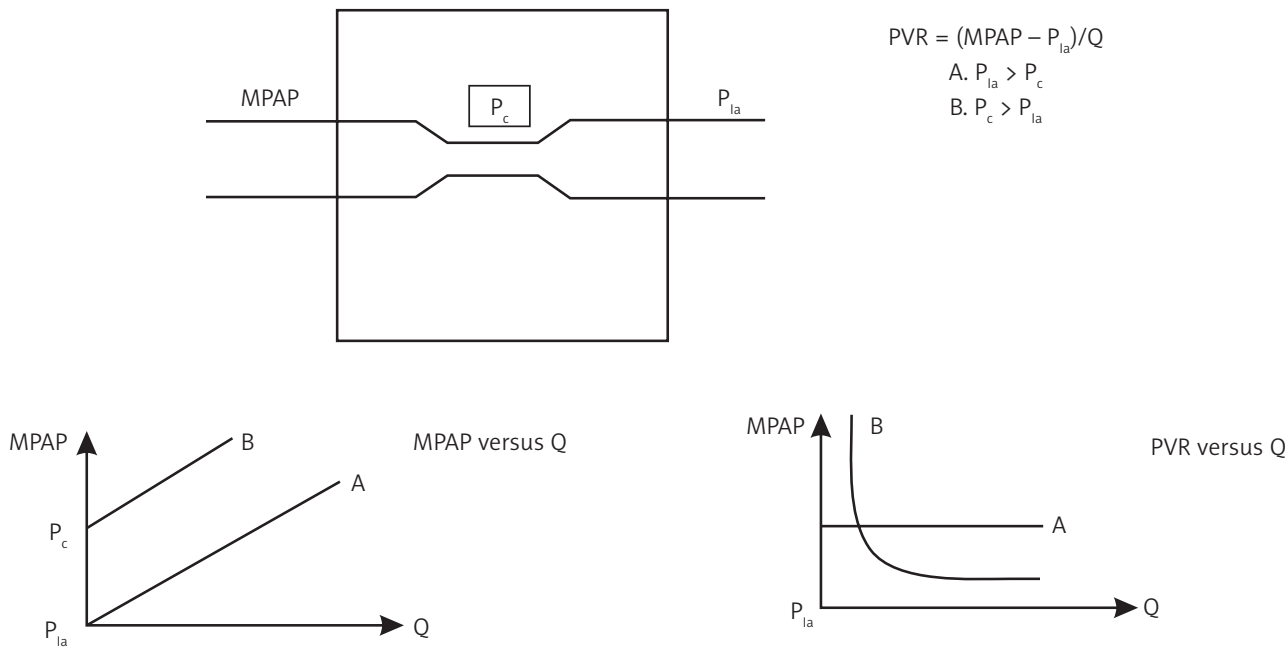

Figure 5. See text for description 


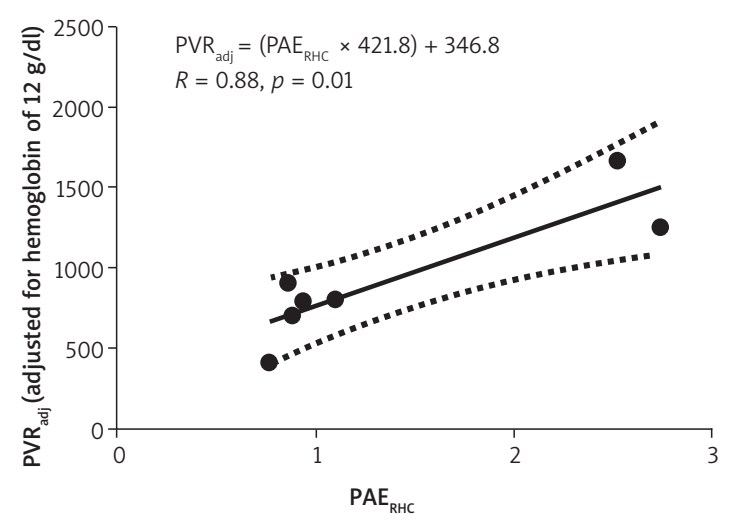

Figure 6. Only the top 7 data points from Figure 2 are included

$P V R_{\text {adj }}$ - pulmonary vascular resistance for hemoglobin of $12 \mathrm{~g} / \mathrm{dl}$ (see text for details). Units: dyne $\times \mathrm{s} / \mathrm{cm}^{5}$. $P A E_{\text {PHC }}$ - pulmonary arterial elastance on right heart catheterization. Units: $\mathrm{mm} \mathrm{Hg} / \mathrm{ml}$. $R$-Pearson coefficient of correlation

(e.g. idiopathic pulmonary arterial hypertension) are encircled with a thicker layer of smooth muscles - resulting in a lower closing pressure. It is possible that the closing pressures in these patients are more consistently lower than the left atrial pressures. In those with the severest forms of pre-capillary pulmonary vascular disease, it is possible that the closing pressures are always lower than the left atrial pressures. Thus, in such patients, PVR becomes a consistent marker of the degree of stiffness of the pulmonary arterial vessels. In such a case, PVR and PAE are expected to have a closer relationship as conceptually PAE represents the stiffness of pulmonary arterial vessels.

Indeed, on the further analysis of RHC-derived PAE and RHC-derived adjusted PVR, the Pearson coefficient of correlation was excellent for the very high PVRs, and was moderate for the lower PVRs (Figures 6, 7).

The uncertainty about the closing pressure may result in entirely misleading PVR values $[13,14]$. Our data point in a similar direction. Questions that our data raise are: Does PVR reliably predict severity of pulmonary vascular disease? Can PAE (RHC-derived or echocardiographic) better predict severity of pulmonary vascular disease?

In patients with PVRs known to be greater than about 9 Wood units (when adjusted for hemoglobin of $12 \mathrm{~g} / \mathrm{dl}$ ), echocardiographic PAE might be accurate enough to predict any changes in PVR as a response to vasodilator therapy (see equation (xii)).

The limitations of our study include a small cohort size, non-simultaneous performance of echocardiograms and $\mathrm{RHCs}$, and retrospective study design. We again recognize that the superior method of selection of patients for this

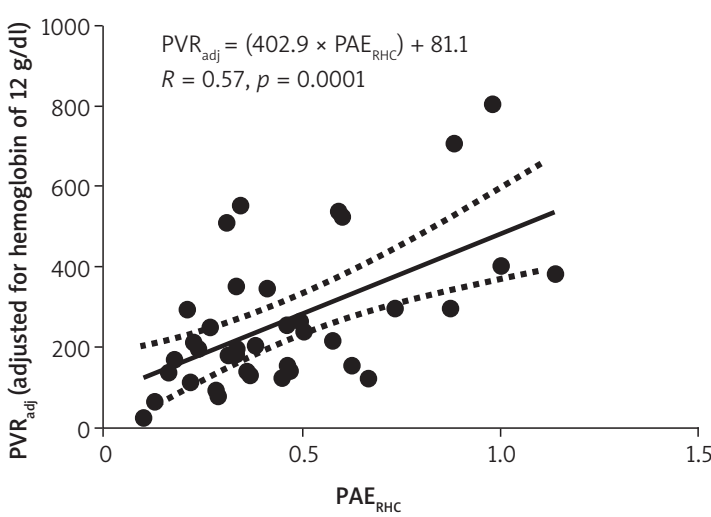

Figure 7. Only the bottom 37 data points from Figure 2 are included

$P V R_{\text {adj }}$ - pulmonary vascular resistance for hemoglobin of $12 \mathrm{~g} / \mathrm{dl}$ (see text for details). Units: dyne $\times \mathrm{s} / \mathrm{cm}^{5}$. $P A E_{R H C}$ - pulmonary arterial elastance on right heart catheterization. Units: $\mathrm{mm} \mathrm{Hg} / \mathrm{ml} . R$ - Pearson coefficient of correlation

retrospective study would be to study all consecutive patients over a time period, and hoping that patients with all ranges of PVRs in adequate numbers would fall in that group. However, we anticipated that the patients who have higher PVR are relatively few due to the rarity of diseases having this state, and that the $\mathrm{RHC}$ registry in our hospital would have a preponderance of patients with low PVR. Anticipating the above-described skewness in the patient selection, we modified the patient selection strategy in the following two ways: 1) we chose, on one hand, only one-third of the large number of patients in our $\mathrm{RHC}$ registry, and 2) we employed, on the other hand, an assortment of recruitment strategies to include a greater number of patients with higher PVR. We believe that these modifications do not affect the validity of our results as our study hypothesis (that RHC-derived PVR and echocardiographic PAE have a linear relationship over a wide range of PVR) should hold true, if it has to, even if a non-consecutive cohort of patients is chosen. Another important limitation of our retrospective study was non-simultaneous performance of echocardiograms and RHCs. Such temporal gaps are unavoidable in routine clinical practice. Faced with a similar question related to study design, Arkles et al. chose a temporal gap of 120 days [15]. Fortunately, the studies done on a vast majority of our patients were separated by only a few days (14 patients on the same day, 12 were separated by 1 day, 11 were separated by 2-7 days, 5 were separated by 8-14 days, and the remaining 3 were separated by 33, 71 and 75 days respectively). The median interval between the paired echocardiogram and RHC was 1 day. The validity of our conclusions is maintained for those patients whose 
A

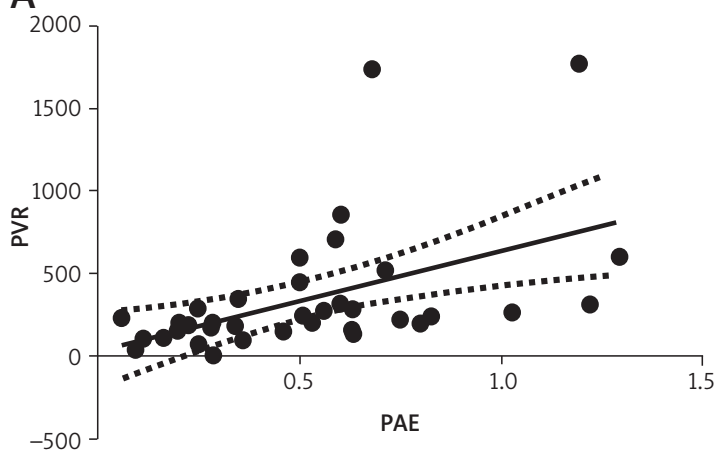

C

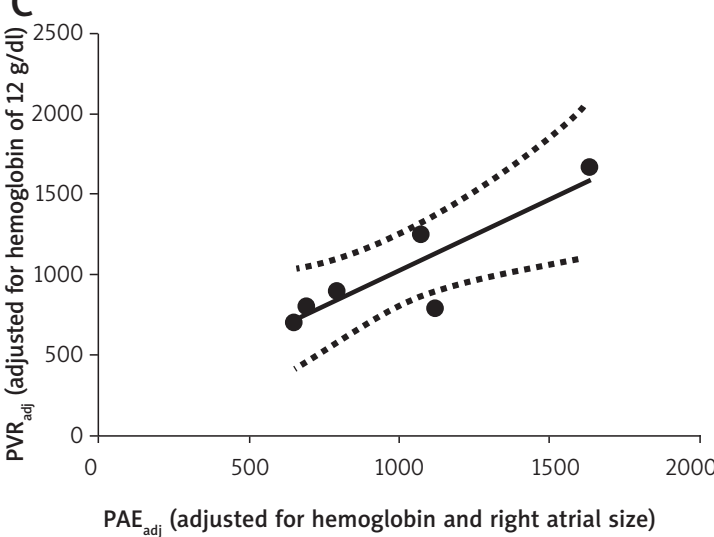

B
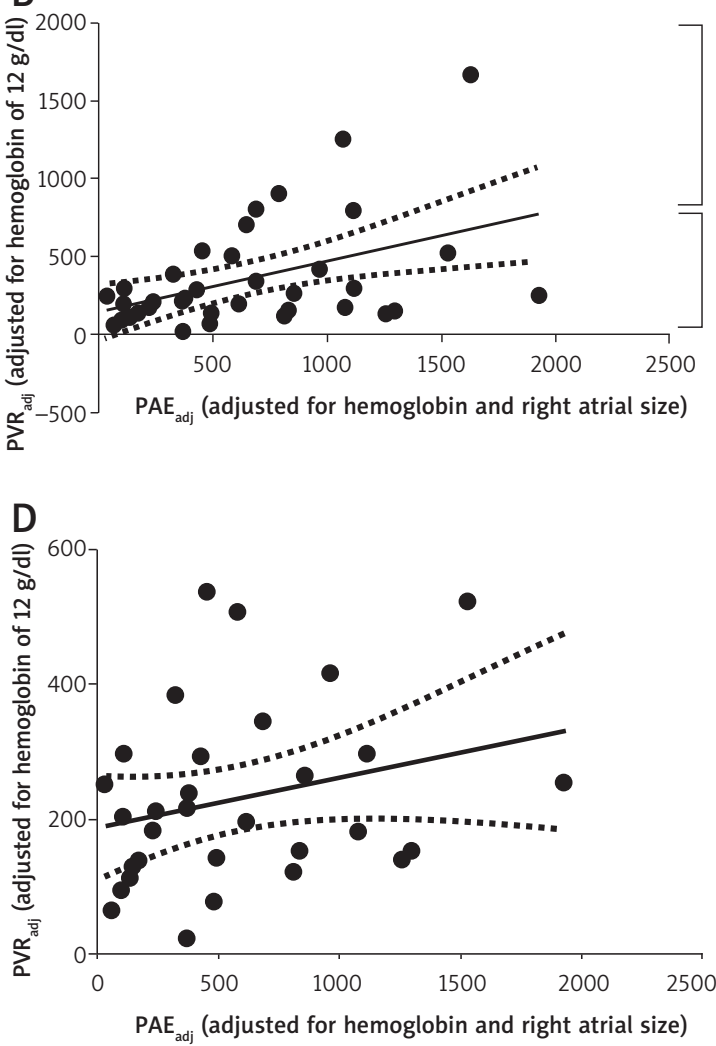

Figure 8. Linear regression analysis between PVR and PAE, or PVR ${ }_{\text {adj }}$ and $P A E_{\text {adj }}$ (when the right heart catheterization and echocardiogram were done within 7 days of each other), $n=37$. A - Linear regression analysis between PVR and PAE: PVR $=603.2 \times$ PAE $+34.3(R=0.49, p=0.002) . \mathrm{B}-$ Linear regression analysis between PVR $\mathrm{R}_{\text {adj }}$ and $\mathrm{PAE}_{\mathrm{d} ; \mathrm{j}}: \mathrm{PVR}_{\mathrm{dj}}=0.326 \times \mathrm{PAE}_{\mathrm{ajj}}+147.4(R=0.45, p=0.005)$. See text for the description of data points depicted by the arrow and the two square brackets. $\mathbf{C}$ - Linear regression analysis between $\mathrm{PVR}_{\mathrm{adj}}$ and PAE ${ }_{\text {adj }}$ Only the top 6 data points as depicted within the top brackets in Figure $8 \mathrm{~B}$ are included: $\mathrm{PVR}_{\mathrm{adj}}=0.882 \times \mathrm{PAE}_{\mathrm{adj}}+148.8(R=0.88$, $p=0.02$ ). Lowest $\mathrm{PVR}_{\mathrm{adj}}$ in this small data set corresponds to 706.0 dyne-s/cm 5 . D - Linear regression analysis between PVR $\mathrm{P}_{\text {adj }}$ and $\mathrm{PAE}_{\text {adj }}$. Only the 31 data points as depicted within the bottom brackets in Figure 8 B are included: $\mathrm{PVR}_{\mathrm{adj}}=0.075 \times \mathrm{PAE}_{\mathrm{adj}}+187.4(R=0.27, p=0.15)$

$P V R$ - pulmonary vascular resistance, PAE - pulmonary arterial elastance, $P V R_{\text {adj }}$ - pulmonary vascular resistance for hemoglobin of $12 \mathrm{~g} / \mathrm{dl}$ (see text for details), $P A E_{\text {adj }}$ - adjusted echocardiographic pulmonary arterial elastance, $R$ - Pearson coefficient of correlation. Units for PVR or PVR adj: dyne $\times \mathrm{s} / \mathrm{cm}^{5}$. Units for PAE or $P A E_{\text {adj: }} \mathrm{mm} \mathrm{Hg} / \mathrm{ml}, R-$ Pearson coefficient of correlation

RHC and echocardiogram had been done within 7 days (Figure 8 , linear regression equations included in the legend). We also recognize that the adjustments for PVR and PAE used in analyzing data are somewhat approximate. However, they are simple enough to be easily tested in further studies.

In conclusion, novel echocardiographic indices have been increasingly studied in recent times to predict clinical outcomes and to assess severity for both left [16] as well as right heart diseases [17]. In our study, we found that echocardiographic PAE did not have a strong linear relationship with RHC-derived PVR over a wide range of PVR. In patients with very advanced pre-capillary pulmonary vascular disease, echocardiographic PAE may be accurate enough to predict any changes in PVR as a response to vasodilator therapy. A prospective study of larger size with simultaneous performance of echocardiograms and RHCs may give definitive answers.

\section{Acknowledgments}

This study was presented in part at the American Thoracic Society Meeting in Philadelphia, Pennsylvania on May 20, 2013.

\section{References}

1. Haddad F, Zamanian R, Beraud AS, et al. A novel non-invasive method of estimating pulmonary vascular resistance in patients with pulmonary arterial hypertension. J Am Soc Echocardiogr 2009; 22: 523-9.

2. Abbas AE, Fortuin FD, Schiller NB, Appleton CP, Moreno CA, Lester SJ. Echocardiographic determination of mean pulmonary artery pressure. Am J Cardiol 2003; 92: 1373-6.

3. Quiñones MA, Otto CM, Stoddard M, Waggoner A, Zoghbi WA. Recommendations for quantification of Doppler 
echocardiography: a report from the Doppler Quantification TaskForce of the Nomenclature and Standards Committee of the American Society of Echocardiography. J Am Soc Echocardiogr 2002; 15: 167-84.

4. Giardini A, Tacy TA. Non-invasive estimation of pressure gradients in regurgitant jets: an overdue consideration. Eur J Echocardiogr 2008; 9: 578-84.

5. Heys JJ, Holyoak N, Calleja AM, Belohlavek M, Chaliki HP. Revisiting the simplified bernoulli equation. Open Biomed Eng J 2010; 4: 123-8.

6. Hatle L, Brubakk A, Tromsdal A, Angelsen B. Noninvasive assessment of pressure drop in mitral stenosis by Doppler ultrasound. Br Heart J 1978; 40: 131-40.

7. Pries AR, Neuhaus D, Gaehtgens P. Blood viscosity in tube flow: dependence on diameter and hematocrit. Am J Physiol 1992; 263: H1770-8.

8. Dawson B, Trapp RG. Basic and clinical biostatistics. 4th ed. The Mcgraw Hill Companies, Inc. 2004

9. Hoffman JI. Pulmonary vascular resistance and viscosity: the forgotten factor. Pediatr Cardiol 2011; 32: 557-61.

10. Linehan JH, Haworth ST, Nelin LD, Krenz GS, Dawson CA. A simple distensible vessel model for interpreting pulmonary vascular pressure-flow curves. J Appl Physiol 1992; 73: 987-94.

11. Rudski LG, Lai WW, Afilolo J, et al. Guidelines for the echocardiographic assessment of the right heart in adults: a report from the American Society of Echocardiography endorsed by the European Association of Echocardiography, a registered branch of the European Society of Cardiology, and the Canadian Society of Echocardiography. J Am Soc Echocardiogr 2010; 23: 685-713.

12. Van den Broeck J, Argeseanu Cunningham S, Eeckels R, Herbst K. Data cleaning: detecting, diagnosing, and editing data abnormalities. PLoS Med 2005; 2: e267.

13. Versprille A. Pulmonary vascular resistance: a meaningless variable. Intensive Care Med 1984; 10: 51-3.

14. Permutt S, Riley RL. Hemodynamics of collapsible vessels with tone: the vascular waterfall. J Appl Physio 1963; 18: 924-32.

15. Arkles JS, Opotowsky AR, Ojeda J, et al. Shape of the right ventricular Doppler envelope predicts hemodynamics and right heart function in pulmonary hypertension. Am J Respir Crit Care Med 2011; 183: 268-76.

16. Możdżan M, Wierzbowska-Drabik K, Kurpesa M, et al. Echocardiographic indices of left ventricular hypertrophy and diastolic function in hypertensive patients with preserved LVEF classified as dippers and non-dippers. Arch Med Sci 2013; 9: 268-75.

17. Mazurek JA, Forfia PR. Enhancing the accuracy of echocardiography in the diagnosis of pulmonary arterial hypertension: looking at the heart to learn about the lungs. Curr Opin Pulm Med 2013; 19: 437-45. 